\title{
Percutaneous coronary intervention in left main disease: SYNTAX, PRECOMBAT, EXCEL and NOBLE-combined cardiology and cardiac surgery perspective
}

\author{
Duk-Woo Park ${ }^{1}$, Jung-Min Ahn ${ }^{1}$, Seung-Jung Park ${ }^{1}$, David P. Taggart ${ }^{2}$ \\ ${ }^{1}$ Department of Cardiology, Asan Medical Center, University of Ulsan College of Medicine, Seoul, Korea; ${ }^{2}$ Nuffield Department of Surgical \\ Sciences, University of Oxford, Oxford, UK \\ Correspondence to: Dr. Seung-Jung Park, MD, PhD. Department of Cardiology, Asan Medical Center, University of Ulsan College of Medicine, Seoul, \\ Korea. Email: sjpark@amc.seoul.kr; David P. Taggart, MD, PhD. Nuffield Department of Surgical Sciences, University of Oxford, Oxford, UK. \\ Email: David.Taggart@ouh.nhs.uk.
}

\begin{abstract}
Although coronary-artery bypass grafting (CABG) is the standard choice of revascularization for significant left main coronary artery (LMCA) disease, percutaneous coronary intervention (PCI) for LMCA disease has been widely expanded with adoption of drug-eluting stents (DES). Several small- and moderate-sized trials of CABG and first-generation DES showed that PCI might be a good alternative for selected patients with LMCA disease. However, these early trials were relatively underpowered and comparative results of contemporary DES and CABG were clearly required. Subsequently, two large-sized trials comparing CABG and contemporary DES (EXCEL and NOBLE) were conducted, but these trials showed conflicting results with regards to the effects of PCI and CABG on clinical outcomes, which raises further uncertainty on the optimal revascularization for LMCA disease. This article serves to summarize the key findings of landmark clinical trials, to share our knowledge and experience and to express personal opinions on current controversies in the treatment of LMCA disease.
\end{abstract}

Keywords: Coronary-artery bypass grafting (CABG); left main coronary artery disease (LMCA disease); percutaneous coronary intervention (PCI)

Submitted Mar 11, 2018. Accepted for publication Mar 31, 2018.

doi: 10.21037/acs.2018.04.04

View this article at: http://dx.doi.org/10.21037/acs.2018.04.04

\section{Introduction}

Owing to the large area of jeopardized myocardium and combined highest ischemic risk, coronary-artery bypass grafting (CABG) has traditionally been the choice of revascularization for significant left main coronary artery (LMCA) disease. In the early period of percutaneous coronary intervention (PCI), due to a high procedural risk and lack of appropriate stent technology and experience, intervention for LMCA disease was only performed as salvage procedure. Over the last two decades, there have dramatic advances in the field of PCI, involving stent devices, techniques, and adjunctive antithrombotic drugs, which lead to substantial improvement in the outcomes of PCI for LMCA disease (1). To establish the potential therapeutic role of PCI as alternative treatment option to
CABG for unprotected LMCA disease, several randomized clinical trials (RCTs) have been conducted. With firstgeneration drug-eluting stents (DES), clinical trials revealed that PCI achieved similar rates of death, but a higher rate of repeat revascularization and a lower rate of stroke (28). With improved efficacy and safety profiles of secondgeneration DES compared to first-generation DES $(9,10)$, more trials confirming the efficacy of contemporary DES were required. Recently the results of two landmark RCTs, the Evaluation of XIENCE Everolimus Eluting Stent Versus Coronary Artery Bypass Surgery for Effectiveness of Left Main Revascularization (EXCEL) and the NordicBaltic-British Left Main Revascularization Study (NOBLE), have been reported $(11,12)$. This perspective article will provide a 'cutting edge' contemporary review of the recent 
trials and speculate on the future direction for optimal LMCA management.

\section{PCI with stenting for LMCA disease}

The introduction of coronary stenting substantially overcame the inherent limitations of balloon angioplasty (i.e., abrupt closure, dissection, or acute recoil) and widened the therapeutic role of PCI for LMCA lesions. In the period of bare-metal stents (BMS), PCI showed acceptable in-hospital and mid-term clinical outcomes among elective low-risk patients (13-17). However, a high risk of angiographic and clinical restenosis hampered the wider expansion of PCI for LMCA disease. With the introduction of DES, that demonstrated superior efficacy with respect to restenosis, the role of PCI for LMCA was expanded considerably and several studies showed favorable short- and long-term clinical results (18-21).

Allied to a revolution in stent technology, improved interventional techniques and adjunctive pharmacotherapy have progressively enhanced PCI outcomes for LMCA disease (1). Second-generation DES employing improved technology and engineering of DES (i.e., novel and thinner strut platforms, easier delivery systems, and biocompatible or bioresorbable polymers) (22). Several clinical studies subsequently reported superior clinical outcomes with second-generation compared to first-generation DES for LMCA PCI (23-25).

In a recent, merged analysis of 4,470 patients with unprotected LMCA disease treated with second-generation DES, there were no significant differences with regard to the 3-year rate of target-vessel failure among different types of second-generation DES including the cobalt-chromium everolimus-eluting stent (CoCr-EES), the biodegradable polymer-biolimus-eluting stent (BP-BES), and the Resolute zotarolimus-eluting stent (Re-ZES) (26). However, the platinum chromium-EES (PtCr-EES) was associated with a higher risk of target-vessel failure than BP-BES (26). The incidence of stent thrombosis was notably low (less than $1.0 \%)$ for all types of DES.

\section{Trials of PCI and CABG for LMCA disease}

\section{First-generation DES: SYNTAX and PRECOMBAT}

Before the EXCEL and NOBLE trials, four RCTs comparing PCI using first-generation DES and CABG were conducted (27): this included SYNTAX patients with
LMCA disease ( $\mathrm{n}=705)$, PRECOMBAT ( $\mathrm{n}=600)$, LEMANS $(\mathrm{n}=100)$ and the report of Boudriot et al. $(\mathrm{n}=201)$. In the LMCA subgroup analysis of the SYNTAX trial $(4,5)$, there were no significant differences with respect to the 5 -year rates of major adverse cardiac and cerebrovascular event (MACCE; 37\% vs. 31\%), mortality (13\% vs. $15 \%$ ) or MI ( $8 \%$ vs. $5 \%$ ) between PCI and CABG. However, the 5 -year rate of repeat revascularization was higher in the PCI group (27\% vs. 16\%) and the rate of stroke was higher in the CABG group ( $2 \%$ vs. $4 \%$ ). In a stratified analysis on the basis of baseline SYNTAX score, the rate of MACCE were similar between PCI and CABG in the lower 2 SYNTAX score tertiles (0-32) (31\% vs. 32\%). In contrast, in the group with high SYNTAX scores ( $\geq 33)$, MACCE occurred significantly more commonly in the PCI than in the CABG group (47\% vs. 30\%).

In the PRECOMBAT trial $(7,8)$, the 5 -year rates of MACCE (18\% vs. $14 \%)$, death (6\% vs. $8 \%)$, MI (2\% vs. $2 \%)$, or stroke (1\% vs. $1 \%)$ were similar between PCI and CABG. However, again, the rate of target-vessel revascularization was significantly higher in the PCI group than in the CABG group (12\% vs. 6\%). However, owing to a wide noninferiority margin and the relatively small number of patients in prior trials using first-generation DES, overall comparative results should be interpreted with caution and cannot be considered definitively clinically directive. This clinical unmet need motivated and underpinned the two large-sized landmark trials of EXCEL and NOBLE.

\section{Second-generation DES: EXCEL and NOBLE}

In discussing and comparing EXCEL and NOBLE it is crucial to recognize similarities and differences with regard to trial design and primary end point. In EXCEL, a total of 1,905 patients with LMCA disease and low or intermediate anatomical complexity (SYNTAX score $\leq 32$ ) were randomly assigned to CABG or PCI with a fluoropolymerbased, CoCr-EES. In NOBLE, 1,201 patients with LMCA disease were randomly assigned to CABG or PCI (11\% of the patients received a first-generation DES and the rest a biolimus-eluting stent). With regards to trial eligibility criteria, NOBLE did not adopt a baseline SYNTAX score as a prespecified inclusion criteria but, instead, excluded patients with more than three additional non-complex coronary lesions or complex additional coronary lesions (length $>25 \mathrm{~mm}$, chronic total occlusion, 2-stent bifurcation, calcified or tortuous vessel morphology). Notably, the primary composite endpoint was different for EXCEL and 
NOBLE: EXCEL adopted the clinically 'harder' endpoint of all-cause death, MI, or stroke, while NOBLE adopted MACCE including all-cause death, non-procedural MI, stroke, or repeat revascularization.

However, the two trials showed conflicting results; EXCEL reported that $\mathrm{PCI}$ is noninferior to $\mathrm{CABG}$ during median follow-up of 3.0 years (IQR, 2.4-3.0), while NOBLE reported that CABG is superior to PCI during median follow-up of 3.1 (IQR, 2.0-5.0). In EXCEL, PCI was noninferior to CABG with respect to the primary composite of death, stroke, or MI at 3 years $(15.4 \% v s$. $14.7 \%)$. The primary end-point events were less common after PCI than after CABG within 30 days ( $4.9 \%$ vs. $7.9 \%$ ). The individual rates of early $\mathrm{MI}$ and major periprocedural adverse events (i.e., bleeding, infection, major arrhythmia, and renal failure) within 30 days were significantly lower with PCI than with CABG $(3.9 \%$ vs. $6.2 \%$ and $8.1 \%$ vs. $23.0 \%$, respectively). In contrast, fewer primary end-point events occurred in the CABG group than in the PCI group between 30 days and 3 years.

An important caveat to EXCEL is that at complete 3-year follow-up, the rate of all-cause mortality tended to be higher in the PCI group than in the CABG group ( $8.0 \%$ vs. $5.8 \%, \mathrm{P}=0.08)$. Consequently, longer clinical follow-up is required to determine whether cross-over in mortality in favor of CABG that just failed to reach statistical significance at 3 years might be significant by 5 years. Furthermore, in contrast to previous trials, there was no difference in the incidence of stroke between PCI and CABG groups (2.3\% vs. $2.9 \%)$. but ischemia-driven revascularization during follow-up was more frequent after PCI than after CABG (12.6\% vs. $7.5 \%)$.

In NOBLE, the 5-year rate of MACCE was significantly higher after PCI than after CABG (29\% vs. 19\%). At 5 years, the rate of death was similar between PCI and CABG $(36 \%$ vs. $33 \%)$, but non-procedural MI (7\% vs. $2 \%)$ and any revascularization $(16 \%$ vs. $10 \%)$ were higher in the PCI group. Unexpectedly, the 5-year of stroke tended to be higher in PCI patients than in CABG patients (7\% vs. 16\%).

Careful interpretation of the differing results from EXCEL and NOBLE is required to minimize the uncertainty on optimal LMCA treatment in the clinical practice. Plausible explanations of conflicting results might be explained by several factors (27). First, the particulars of clinical practice in the participating sites as well as the specific expertise of the interventional cardiologists and cardiac surgeons who performed the procedures might influence the comparative outcomes after LMCA revascularization. In the trials process, an integrated and skilled heart team approach was evident in EXCEL, but not in NOBLE. Second, differences in population size and follow-up might influence the conflicting results as the benefits of CABG tend to become more apparent with longer follow-up. In the NOBLE, there was an interim change in protocol and primary outcome reporting with extension of follow-up owing to lower than expected MACCE rates, which might question the trial integrity and internal validation of NOBLE. Third, EXCEL and NOBLE used different stent platforms. EXCEL used a thin-strut, fluoropolymer-based CoCr-EES, which was associated with the lowest risk of stent thrombosis of all available DES (28). By contrast, NOBLE used firstgeneration, thicker-strut, stainless-steel, sirolimus-eluting Cypher stent $(11 \%)$ or the biolimus-eluting Biomatrix Flex stent $(89 \%)$. There was a substantial difference in rates of definite stent thrombosis $(0.7 \%$ in the EXCEL and $3 \%$ in the NOBLE). Fourth, the NOBLE trials included the softer clinical endpoint of repeat revascularization as part of the primary composite endpoint. Thus, conflicting primary results between the EXCEL and NOBLE trials were mainly driven by the differing definition of the primary composite endpoint. It has been long debated whether the risk of repeat revascularization can be equally balanced against the risk of death, MI, or stroke. One previous trial reported that the increase in the rate of repeat revascularization with PCI did not translate into an increase in the rate of death or MI (29). Fifth, due to inter-trial heterogeneity for the definition of MI, overall trial results could vary widely between EXCEL and NOBLE. In EXCEL, clinically relevant MI definition by the SCAI was used (30), but periprocedural MI was disregarded in NOBLE. Because a uniform definition of MI that does not 'penalize' either revascularization approach is still lacking, further studies are required to enhance standardization of $\mathrm{MI}$ definition for clinical trials comparing PCI and CABG. Finally, unexpectedly, the 5 -year risk of stroke was more than twice higher after PCI rather than after CABG in NOBLE, which was the opposite to the results of EXCEL where there was no significant difference in the incidence of stroke. Because a greater rate of late stroke after PCI in NOBLE lacks a clear explanation of biologic plausibility this result might be most likely due to a chance effect (31).

\section{Updated meta-analysis of PCI and CABG}

Numerous RCTs have compared CABG with PCI for 
patients with multivessel or LMCA disease. However, no studies have been individually powered to detect a difference in mortality between the revascularization strategies. A recent large, collaborative, pooled analysis of individual patient data reported a comparable treatment effect of PCI and $\mathrm{CABG}$ with regard to all-cause mortality up to 5 years in selected patients in RCTs (32). This report included 11 RCTs involving 11,518 patients selected by heart teams who were assigned to PCI $(n=5,753)$ or to CABG $(n=5,765)$. The 5-year rate of all-cause mortality was $11.2 \%$ after PCI and 9.2\% after CABG (HR 1.20, 95\% CI, 1.06-1.37; $\mathrm{P}=0.004)$. Interestingly, 5-year all-cause mortality was significantly different between the interventions in patients with multivessel disease (11.5\% after PCI vs. $8.9 \%$ after CABG; HR 1.28, 95\% CI, 1.09-1.49; $\mathrm{P}=0.002)$, including in those with diabetes $(15.5 \%$ vs. $10.0 \% ; 1.48,1.19-1.84$; $\mathrm{P}=0.0004)$, but not in those without diabetes $(8.7 \%$ vs. $8.0 \%$; $1.08,0.86-1.36 ; \mathrm{P}=0.49)$. By contract, the 5 -year rate of allcause mortality was similar between the interventions in patients with LMCA disease (10.7\% after PCI vs. $10.5 \%$ after CABG; 1.07, 0.87-1.33; $\mathrm{P}=0.52$ ), regardless of diabetes status and SYNTAX score. In summary of this metaanalysis, CABG had a mortality benefit over PCI in patients with multivessel disease, particularly those with diabetes and higher coronary complexity. No benefit for CABG over PCI was seen in patients with LMCA disease. An important caution for LMCA disease is that with few exceptions the patients had been more highly selected than those seen in routine practice.

\section{Perspective and summary}

Although recent RCTs showed favorable outcomes of PCI comparable to CABG, some important points should be considered. First, while the results of PCI vs. CABG are encouraging, these results in highly selected trial cohorts may not be fully applicable to a diversity of patients or clinical circumstances encountered in daily practice (i.e., "all-comer" patients). Second, as noted in EXCEL and NOBLE, a relative treatment effect of PCI and CABG was substantially different over time and especially noting a late catch-up effect. Until recently, long-term follow-up studies up to 5 to 10 years for LMCA revascularization are still limited $(33,34)$. Limited follow-up could have penalized the CABG group, because the long-term benefits of CABG over PCI have not typically been fully evident until 5 to 10 years after the revascularization. Trial patients in EXCEL and NOBLE will be followed up at 5 and 10 years, which will add additional valuable information on long-term results. Third, in the contemporary "real-world" practice, although clinical equipoise was present for either PCI or CABG, patients with less complex clinical and anatomic characteristics (i.e., isolated left main disease, ostial or shaft left main disease, or additional non-complex single- or two-vessel disease) may be considered for PCI or CABG. However, although PCI with contemporary DES is now more widely considered for a broader range of anatomical complexity, further studies are required to determine whether PCI is an acceptable alternative to CABG in patients with high anatomical complexity in LMCA disease.

On the basis of cumulative evidence of comparative effectiveness studies of LMCA revascularization for selected patients, at least over the medium term, guideline recommendations for LMCA PCI have become less stringent over time (1). Although a dramatic change in class of recommendation for LMCA PCI is not anticipated, EXCEL and NOBLE provide additional, but conflicting, evidence that may influence current guidelines. Ideally, proposal of less restrictive PCI indications should be based on at least 5 -year follow-up. Thus, it might be more cautious about treatment recommendations until the 5 -year outcomes of full-cohort patients enrolled EXCEL and NOBLE are available. Also, given that the SYNTAX score was not an important factor in guiding decision-making for optimal revascularization and to differentiate the comparative outcomes between CABG and PCI in EXCEL and NOBLE, whether the SYNTAX score can work as the pivotal factor in the future revascularization guidelines needs further discussion.

In summary, the recent report of EXCEL and NOBLE trials add to the level of evidence for optimal management of LMCA disease in selected patients but with conflicting evidence, and cannot, yet, definitively reposition the therapeutic role of each revascularization approach. However, there might currently be no clearcut (all-or-none) definitive answer regarding the optimal revascularization strategy. The Heart Team approach has a very relevant role in guiding individual patient decisionmaking and for patient-centered care. In the interest of truly informed consent, both options of CABG and PCI should be discussed with the patient. Thus, the optimal choice of revascularization modality recommendation for LMCA disease should be made after discussion among the heart team members by determining the general appropriateness and eligibility of PCI or CABG and, additionally, taking into account the specific circumstances of each patient and 
individual preferences (27).

\section{Acknowledgements}

None.

\section{Footnote}

Conflicts of Interest: The authors have no conflicts of interest to declare.

\section{References}

1. Lee PH, Ahn JM, Chang M, et al. Left main coronary artery disease: secular trends in patient characteristics, treatments, and outcomes. J Am Coll Cardiol 2016;68:1233-46.

2. Buszman PE, Kiesz SR, Bochenek A, et al. Acute and late outcomes of unprotected left main stenting in comparison with surgical revascularization. J Am Coll Cardiol 2008;51:538-45.

3. Buszman PE, Buszman PP, Banasiewicz-Szkrobka I, et al. Left main stenting in comparison with surgical revascularization: 10-year outcomes of the (left main coronary artery stenting) LE MANS Trial. JACC Cardiovasc Interv 2016;9:318-27.

4. Morice MC, Serruys PW, Kappetein AP, et al. Outcomes in patients with de novo left main disease treated with either percutaneous coronary intervention using paclitaxeleluting stents or coronary artery bypass graft treatment in the Synergy Between Percutaneous Coronary Intervention with TAXUS and Cardiac Surgery (SYNTAX) trial. Circulation 2010;121:2645-53.

5. Morice MC, Serruys PW, Kappetein AP, et al. Five-year outcomes in patients with left main disease treated with either percutaneous coronary intervention or coronary artery bypass grafting in the synergy between percutaneous coronary intervention with taxus and cardiac surgery trial. Circulation 2014;129:2388-94.

6. Boudriot E, Thiele H, Walther T, et al. Randomized comparison of percutaneous coronary intervention with sirolimus-eluting stents versus coronary artery bypass grafting in unprotected left main stem stenosis. J Am Coll Cardiol 2011;57:538-45.

7. Park SJ, Kim YH, Park DW, et al. Randomized trial of stents versus bypass surgery for left main coronary artery disease. N Engl J Med 2011;364:1718-27.

8. Ahn JM, Roh JH, Kim YH, et al. Randomized Trial of
Stents Versus Bypass Surgery for Left Main Coronary Artery Disease: 5-Year Outcomes of the PRECOMBAT Study. J Am Coll Cardiol 2015;65:2198-206.

9. Bangalore S, Kumar S, Fusaro M, et al. Short- and longterm outcomes with drug-eluting and bare-metal coronary stents: a mixed-treatment comparison analysis of 117 762 patient-years of follow-up from randomized trials. Circulation 2012;125:2873-91.

10. Palmerini T, Benedetto U, Biondi-Zoccai G, et al. Longterm safety of drug-eluting and bare-metal stents: evidence from a comprehensive network meta-analysis. J Am Coll Cardiol 2015;65:2496-507.

11. Stone GW, Sabik JF, Serruys PW, et al. EverolimusEluting Stents or Bypass Surgery for Left Main Coronary Artery Disease. N Engl J Med 2016;375:2223-35.

12. Makikallio T, Holm NR, Lindsay M, et al. Percutaneous coronary angioplasty versus coronary artery bypass grafting in treatment of unprotected left main stenosis (NOBLE): a prospective, randomised, open-label, non-inferiority trial. Lancet 2016;388:2743-52.

13. Park SJ, Park SW, Hong MK, et al. Stenting of unprotected left main coronary artery stenoses: immediate and late outcomes. J Am Coll Cardiol 1998;31:37-42.

14. Silvestri M, Barragan P, Sainsous J, et al. Unprotected left main coronary artery stenting: immediate and mediumterm outcomes of 140 elective procedures. J Am Coll Cardiol 2000;35:1543-50.

15. Park SJ, Hong MK, Lee CW, et al. Elective stenting of unprotected left main coronary artery stenosis: effect of debulking before stenting and intravascular ultrasound guidance. J Am Coll Cardiol 2001;38:1054-60.

16. Black A, Cortina R, Bossi I, et al. Unprotected left main coronary artery stenting: correlates of midterm survival and impact of patient selection. J Am Coll Cardiol 2001;37:832-8.

17. Takagi T, Stankovic G, Finci L, et al. Results and longterm predictors of adverse clinical events after elective percutaneous interventions on unprotected left main coronary artery. Circulation 2002;106:698-702.

18. Park SJ, Kim YH, Lee BK, et al. Sirolimus-eluting stent implantation for unprotected left main coronary artery stenosis: comparison with bare metal stent implantation. J Am Coll Cardiol 2005;45:351-6.

19. Valgimigli M, van Mieghem CA, Ong AT, et al. Shortand long-term clinical outcome after drug-eluting stent implantation for the percutaneous treatment of left main coronary artery disease: insights from the RapamycinEluting and Taxus Stent Evaluated At Rotterdam 
Cardiology Hospital registries (RESEARCH and T-SEARCH). Circulation 2005;111:1383-9.

20. Chieffo A, Park SJ, Valgimigli M, et al. Favorable longterm outcome after drug-eluting stent implantation in nonbifurcation lesions that involve unprotected left main coronary artery: a multicenter registry. Circulation 2007;116:158-62.

21. Chieffo A, Stankovic G, Bonizzoni E, et al. Early and mid-term results of drug-eluting stent implantation in unprotected left main. Circulation 2005;111:791-5.

22. Stefanini GG, Holmes DR Jr. Drug-eluting coronaryartery stents. N Engl J Med 2013;368:254-65.

23. Valenti R, Migliorini A, Parodi G, et al. Clinical and angiographic outcomes of patients treated with everolimus-eluting stents or first-generation Paclitaxeleluting stents for unprotected left main disease. J Am Coll Cardiol 2012;60:1217-22.

24. Kim YH, Park DW, Ahn JM, et al. Everolimus-eluting stent implantation for unprotected left main coronary artery stenosis. The PRECOMBAT-2 (premier of randomized comparison of bypass surgery versus angioplasty using sirolimus-eluting stent in patients with left main coronary artery disease) study. JACC Cardiovasc Interv 2012;5:708-17.

25. Buchanan GL, Chieffo A, Bernelli C, et al. Two-year outcomes following unprotected left main stenting with first vs. new-generation drug-eluting stents: the FINE registry. EuroIntervention 2013;9:809-16.

26. Lee PH, Kwon O, Ahn JM, et al. Safety and Effectiveness of Second-Generation Drug-Eluting Stents in Patients With Left Main Coronary Artery Disease. J Am Coll Cardiol 2018;71:832-41.

27. Park DW, Park SJ. Percutaneous Coronary Intervention of Left Main Disease: Pre- and Post-EXCEL (Evaluation of XIENCE Everolimus Eluting Stent Versus Coronary Artery Bypass Surgery for Effectiveness of Left Main Revascularization) and NOBLE (Nordic-Baltic-British

Cite this article as: Park DW, Ahn JM, Park SJ, Taggart DP. Percutaneous coronary intervention in left main disease: SYNTAX, PRECOMBAT, EXCEL and NOBLE-combined cardiology and cardiac surgery perspective. Ann Cardiothorac Surg 2018;7(4):521-526. doi: 10.21037/acs.2018.04.04
Left Main Revascularization Study) Era. Circ Cardiovasc Interv 2017;10.

28. Palmerini T, Biondi-Zoccai G, Della Riva D, et al. Stent thrombosis with drug-eluting and bare-metal stents: evidence from a comprehensive network meta-analysis. Lancet 2012;379:1393-402.

29. Serruys PW, Morice MC, Kappetein AP, et al.

Percutaneous coronary intervention versus coronary-artery bypass grafting for severe coronary artery disease. N Engl J Med 2009;360:961-72.

30. Moussa ID, Klein LW, Shah B, et al. Consideration of a new definition of clinically relevant myocardial infarction after coronary revascularization: an expert consensus document from the Society for Cardiovascular Angiography and Interventions (SCAI). J Am Coll Cardiol 2013;62:1563-70.

31. Mack M, Holmes DR. Randomised trials in left main disease: a NOBLE effort. Lancet 2016;388:2715-6.

32. Head SJ, Milojevic M, Daemen J, et al. Mortality after coronary artery bypass grafting versus percutaneous coronary intervention with stenting for coronary artery disease: a pooled analysis of individual patient data. Lancet 2018;391:939-48.

33. Park DW, Seung KB, Kim YH, et al. Long-term safety and efficacy of stenting versus coronary artery bypass grafting for unprotected left main coronary artery disease: 5-year results from the MAIN-COMPARE (Revascularization for Unprotected Left Main Coronary Artery Stenosis: Comparison of Percutaneous Coronary Angioplasty Versus Surgical Revascularization) registry. J Am Coll Cardiol 2010;56:117-24.

34. Park DW, Kim YH, Yun SC, et al. Long-term outcomes after stenting versus coronary artery bypass grafting for unprotected left main coronary artery disease: 10-year results of bare-metal stents and 5-year results of drug-eluting stents from the ASANMAIN (ASAN Medical Center-Left MAIN Revascularization) Registry. J Am Coll Cardiol 2010;56:1366-75. 